\title{
FUNDAMENTOS PARA EL ESTUDIO DE LAS CORRIENTES DE INVESTIGACIÓN EN CONTABILIDAD
}

\author{
FUNDAMENTALS FOR THE STUDY OF RESEARCH FLOWS IN ACCOUNTING \\ JUSTIFICATIVA PARA O ESTUDO DA PESQUISA ATUAL EM CONTABILIDADE
}

Por: VILLARREAL_ José Luis, CÓRDOBA MARTÍNEZ _ Jorge Xavier

\begin{abstract}
Magister en Gestión Empresarial, Universidad Libre, seccional Cali. Contador Público, Universidad Mariana, Pasto. Docente-Investigador de tiempo completo del programa de contaduría pública de la Universidad Mariana. Integrante del Grupo de Investigación Identidad Contable. Docente Hora Cátedra programa de contaduría pública de la Universidad de Nariño. Email: joseluisvillarreal.di@gmail. com, Colombia.
\end{abstract}

Especialista en Pedagogía e Investigación en la Educación Superior, y Contador Público, Universidad Mariana, Pasto. Estudiante del Doctorado en Ciencias Contables, Universidad de los Andes, Venezuela. Docente-Investigador de tiempo completo del programa de contaduría pública de la Universidad Mariana. Integrante del Grupo de Investigación Identidad Contable. Email: xaviercordoba@yahoo.es, Colombia.

Recibido: 8 de julio de 2016

Aprobación definitiva: 21 de mayo de 2017

DOI: http://dx.doi.org/10.22267/rtend.171802.81

\section{RESUMEN}

La contabilidad como disciplina de conocimiento se alimenta de teorías e investigaciones que permiten ampliar sus postulados y argumentos en la vía de su fundamentación que a la vez impacta en su ejercicio práctico. Así mismo, la evolución del conocimiento contable presenta avances desde la corriente principal y la denominada contabilidad emergente representada por la investigación contable crítica y la investigación contable interpretativa.

De lo anterior se desprende la necesidad de reflexionar sobre las categorías de las corrientes contables: principal (CP), interpretativa (CI) y crítica (CC), con el fin de dar respuesta a la pregunta ¿Cuáles son los fundamentos para el estudio de las corrientes de investigación en contabilidad? El diseño metodológico del artículo, adopta un enfoque descriptivo y reflexivo a partir de una revisión documental sobre avances interdisciplinarios de la contabilidad. Como conclusión principal se muestran diferencias en el andamiaje de las tres corrientes en estudio; así la 
contabilidad no es reflejo automático de asuntos económicos, por el contrario responde a condiciones históricas, e intereses ideológicos y políticos de distintos grupos empresariales, estatales, académicos y sociales.

Palabras clave: corriente contable principal, corriente contable interpretativa, corriente contable crítica e investigación contable.

JEL: M21, M41.

\begin{abstract}
Accounting as a discipline of knowledge is nourished by theories and investigations that allow to expand its postulates and arguments in the way its foundation that at the same time impacts on its practical exercise. Likewise, the evolution of accounting knowledge presents advances from the mainstream and so-called emerging accounting represented by crítical accounting research and interpretive accounting research.
\end{abstract}

From the above, it is necessary to reflect on the categories of accounting currents: principal (CP), interpretative (CI) and critic (CC), in order to answer the question What are the fundamentals for the study of The currents of research in accounting? The methodological design of the article adopts a descriptive and reflective approach based on a documentary review of interdisciplinary accounting advances. The main conclusion shows differences in the scaffolding of the three streams under study; Thus accounting is not an automatic reflection of economic matters, but rather responds to historical conditions, ideological and political interests of different business, state, academic and social groups.

Keywords: main accounting current, interpretive accounting current, crítical accounting current and accounting research.

JEL: M21, M41.

\title{
RESUMO
}

A contabilidade como uma disciplina de conhecimento alimenta de teorias e pesquisas que expandem suas premissas e argumentos na forma como a sua fundação, que em impactos ligar o seu exercício prático. Da mesma forma, a evolução do conhecimento contábil apresenta avanços do mainstream e os chamados pesquisa em contabilidade contabilidade emergente representado pela crítica e pesquisa em contabilidade interpretativa.

Precede a necessidade de refletir sobre as categorias de correntes de contabilidade segue: principal (CP), interpretação (CI) e crítico (CC), a fim de responder à pergunta: Quais são os elementos básicos para estudar pesquisas atuais em contabilidade? 0 desenho metodológico do artigo adota uma abordagem descritiva e reflexiva a partir de uma revisão da literatura sobre os avanços interdisciplinares em contabilidade. As principais diferenças conclusão são mostrados no andaime dos três fluxos em estudo; e contabilidade não é reflexo automático de assuntos econômicos, por outro 
lado, responde a condições históricas e aspectos ideológicos e políticos de vários grupos empresariais, estaduais, acadêmicos e interesses sociais.

Palavras-chave: corriente contable principal, corriente contable interpretativa, corriente contable crítica e investigación contable.

JEL: M21, M41.

\section{INTRODUCCIÓN}

La teoría e investigación contable son el centro de estudio del texto en donde se busca presentar la evolución del conocimiento contable a partir de sus avances de investigación en la corriente principal, al tiempo realizar una presentación de la denominada contabilidad emergente representada por la investigación contable crítica y la investigación contable interpretativa.

Se conoce que la teoría tiene como propósito explicar y fundamentar el conocimiento en particular, por tanto es insumo de toda ciencia y disciplina, de esta forma "reflexionar sobre la teoría, así como consultar los desarrollos teóricos, es un reto motivante para quienes deseen hacerse partícipe de las grandes revoluciones que se viven desde el pensamiento" (Machado y Viloria, 2016:270).

De igual forma, las reflexiones teóricas y epistemológicas permiten repensar y deconstruir el conocimiento con el fin de ampliar sus fronteras, este camino no fácil de recorrer, requiere de posturas críticas y capacidad de análisis, así los pioneros en estudios sobre investigación interpretativa y crítica en contabilidad como los profesores Baker y Bettner (1980:296) manifiestan"creemos que la investigación académica debe ser abordada con una mente abierta que permite a los investigadores buscar nuevos conocimientos sin riesgo de ostracismo o castigo".

En consecuencia, estos párrafos son una invitación a la reflexión en torno a la evolución del pensamiento contable en sus diferentes enfoques teóricos, metodológicos, y las corrientes funcionalistas y emergentes (interpretativa y crítica) que conforman, para sus autores la nueva teoría e investigación contable.

El documento se estructura en tres apartados, inicia con una breve introducción; posteriormente en un primer capítulo se establece la metodología utilizada en su construcción; seguidamente en un segundo capítulo, se presentan los fundamentos sobre las corrientes: principal, interpretativa y crítica en contabilidad; en un tercer y último capítulo, se realiza una categorización de las corrientes de investigación en contabilidad; y finalmente se plantean unas conclusiones iniciales.

\section{METODOLOGÍA}

El diseño metodológico del artículo, adopta un enfoque descriptivo y reflexivo a partir de una revisión documental sobre avances interdisciplinarios de la contabilidad. Las fuentes 
de información son bibliográficas, constituidas por textos relacionados con fundamentos de las corrientes de investigación en contabilidad.

\section{FUNDAMENTOS SOBRE LAS CORRIENTES: PRINCIPAL, INTERPRETATIVA Y CRÍTICA EN CONTABILIDAD}

A partir de los años 70's los contadores académicos presentaron síntomas de preocupación sobre el estado y desarrollo de la investigación contable; esto porque "no había una teoría completamente aceptada sobre la forma de producir la información contable externa" (Chua, 1986: 38), a partir de esto surgieron diferentes teorías (paradigmas) como guías para la formulación de políticas, las cuales terminaron creando varias escuelas de contabilidad, sin alcanzar un consenso en una sola teoría contable.

Asimismo, algunos académicos de corriente crítica (Hopwood, 1984; Burchell et al, 1980; Tinker, 1980) intentaron publicar los resultados de sus investigaciones contables con enfoque interdisciplinario y crítico en las revistas emblemáticas de la academia tradicional (The Accounting Review, Accounting Horizons), esto generó el origen de unas nuevas corrientes de investigación contable, conocidas como la corriente interpretativa y crítica, las cuales crearon sus propias revistas para la difusión de sus investigaciones (Accounting, Organitations and Society, Crítical Perpectives on Accounting, y Accounting, Auditing and Accountability Journal).

A continuación de describen los aspectos fundamentales de las tres corrientes de investigación: principal, interpretativa y crítica.

\section{III.1 Corriente principal}

Respecto a la corriente principal de la contabilidad, interesada principalmente del funcionamiento de la contabilidad financiera y vinculada con la regulación - normas (Ryan; Scapens y Theobald, 2004), así en la investigación clásica en contabilidad "se conoce como corriente principal, la cual ha producido importantes resultados en pro del beneficio de los usuarios de la información contable financiera (Giraldo, 2008:77).

Conste pues que en esta corriente, como afirman Ryan; Scapens y Theobald (2004:59), "los trabajos (investigación contable) parten de una visión objetiva de la sociedad, consideran la conducta individual como determinística y usan la observación empírica y una metodología positiva de la investigación", efectivamente en la corriente principal se ha transitado de tradicionales escuelas patrimonialistas a nuevas escuelas como las de información, del control y neo patrimonialista, con visión positivista y pragmática, el uso de métodos cuantitativos y de carácter empírico - utilitarista de la información contable (Gómez, 2004).

La corriente principal ha sido objeto de revisión y construcción de modelos contables que en la actualidad predominan en el mundo: a) modelo principios de contabilidad generalmente aceptados - gaap emitido por Financial Accounting Standard Board - FASB y b) el modelo europeo que formula los estándares internacionales de información financiera - ifrs emitido por International Accounting Standard Board - IASB. 
A esto se añade que de los modelos contables indicados se materializaron en la práctica mediante el proceso contable y la presentación de estados financieros. Respecto al proceso contable y generación de información financiera útil en la toma de decisiones económicas, afirma Franco (2015:176), que: "las dimensiones relacionadas con técnica contable (el proceso de construir información), regulación contable (el conjunto de reglas que determinan la construcción de una determinada información) y métodos contables (la forma de construir las reglas)" así, se profundiza en un enfoque economicista vinculado con el modelo y sistema contable internacional que busca una visión "objetivista del mundo" con interés por la regulación (normalización de prácticas contables y en auditoria).

Por lo tanto, en la corriente principal, la contabilidad presenta relación con otras disciplinas, las construcciones como la partida doble tienen un componente formal de carácter aritmético (Salgado 2015), denota relaciones entre esta y las disciplinas formales como la matemática. Sin embargo toma fuerza la relación con las ciencias económicas, con la cual se comparten distintas variables como: circulación de la riqueza, procesos de optimización de recursos, maximización de beneficios a nivel micro y macro, hacienda y fiscalidad, todos estos procesos están ligados a la contabilidad por su enfoque utilitarista y técnico, reconocimiento, valoración, representación y revelación con el fin de presentar hechos económicos relacionados con la rentabilidad, creación o destrucción de riqueza material.

En este sentido, desde un enfoque interdisciplinario (la contabilidad) busca estudiar y comprender la realidad que al utilizar herramientas tecnológicas generan avance, modernizan lo contable y los procesos de generación de información, que capturan datos en tiempo real y permiten elaborar informes cuantitativos y cualitativos, que una vez interpretados y analizados son útiles para una mejor toma de decisiones (Castillo, Córdoba y Villarreal, 2008:24).

Ahora es oportuno insistir como la corriente principal no tiene como objetivo el intento de evaluar o posibilidad de cambiar una estructura institucional (el esquema actual de gobierno, los mercados, los precios y formas organizacionales) (Chua,1986); en cambio promueve procesos de regulación contable, a tal punto de existir organismo considerados reguladores globales de la información contable y estándares para la preparación de estados financieros (tanto para el mercado de capitales como para pymes y entidades sin ánimo de lucro - IASB).

El modelo de contable del IASB, abarca múltiples organizaciones (entidades), no solo empresas o grupos económicos que cotizan en bolsa; pese a ello, el enfoque explícito del modelo contable planteado por el IASB da prevalencia a la producción de información para inversores, acreedores y prestamistas, actuales y potenciales, que actúan en mercados financieros Gómez (2016:5).

Así se demuestra el enfoque funcionalista de la corriente dominante en contabilidad. 
Otra característica relevante respecto a la aplicación de esta corriente, es como la contabilidad emplea tecnologías de medición, reportes, auditoría y vigilancia y con esto contribuye a expandir la mentalidad de mercado y la gobernanza (Giraldo, 2008), acompañado de procesos de captación, medición y valoración de la circulación interna, así como su racionalización y control, con el fin de suministrar a la organización la información relevante para la toma de decisiones empresariales.

Desde la aplicación práctica, de la corriente principal en contabilidad se presentan los servicios que ofrecen empresas de contadores, auditores y asesores, en especial a firmas de servicios profesionales globalizadas, las cuales, "se presenta como agentes del imperialismo contemporáneo, que reproducen las relaciones centro-periferia, las desigualdades globales y las relaciones de poder al estilo colonial, en un mundo supuestamente post-imperial" (Giraldo y Flor, 2015: 450).

\section{III.2 Corriente interpretativa}

Frente a los planteamientos de la corriente principal y la corriente crítica, (fundamentada en la teoría crítica de Habermas y la escuela de Francfort, promueve la capacidad de cuestionamiento de la legitimidad política de los diversos regímenes de contabilidad, con el fin de involucrarse en el control social y los conflictos del poder económico e impacto en la humanidad: desempleo, equidad y redistribución de la riqueza), surgió una nueva corriente alternativa denominada investigación contable interpretativa (ICI) desarrollada en los años ochenta del siglo veinte en Reino Unido.

Esta se inicia con la creación de la revista Accounting Organizations and Society en 1976, liderada por el profesor Anthonny G. Hopwood. Esta corriente, está fundamentada en autores posmodernos como Foucault, Latour Bourdieu, Habermas, Giddens y Weber, cuyo propósito es describir, comprender e interpretar los significados que actores humanos aplican a los símbolos y a las estructuras (Macías, 2013).

La ICI se caracteriza inicialmente porque no está interesada en la resolución de problemas, su objetivo es "comprender los fenómenos de interacción entre personas en situaciones sociales y su fundamento filosófico es que el comportamiento humano es completamente ajeno a los fenómenos físicos" (Larrinaga, 1999:113). En ese sentido, su propósito es conocer, no es actuar, o dicho de otra forma, no busca procesos de intervención, solo de interpretación y comprensión de los problemas.

En esta corriente, la realidad social es una construcción humana, es decir, busca comprender un hecho social acudiendo e interpretando a sus actores. Por lo tanto, los problemas siempre estarán inmersos en esa realidad, así las cosas, el investigador interpretativo tratara de estudiar su naturaleza como un fenómeno más de la realidad social a investigar (Larrinaga, 1999).

De lo anterior, se puede deducir que los esquemas interpretativos son esencialmente sociales e inter subjetivos (Macias, 2016), pues la alternativa interpretativa busca darle 
sentido a las acciones humanas, cada acción humana tiene un propósito, tiene un mundo de significados predeterminados, arraigada en experiencias pasadas.

La misión fundamental de la ICI es comprender la práctica contable en los contextos donde se desarrollan, lo cual permite darle sentido a dicha práctica. Por su parte, el instrumento de investigación que se maneja es el lenguaje y la conversación, que se utiliza como texto donde el investigador interpretativo presupone que existe cierto significado y aspectos de teoría y praxis que merecen ser comprendidos (Larrinaga, 1999).

El profesor Giraldo (2008:85), en esta corriente contable plantea que:

En diferentes situaciones del sistema de información contable se logra identificar que su funcionamiento responde a motivaciones e intereses ya hoy bien definidos e identificados, en donde es posible encontrar el significado de los motivos de los agentes que hacen del sistema contable una estrategia en la dinámica organizacional.

En síntesis, un reto fundamental para los investigadores contables interpretativos es "mantener su identidad en medio de la corriente crítica y la corriente principal; esto se puede lograr mediante la construcción colectiva de conocimiento contable interpretativo, mediante redes de trabajo internacionales e interdisciplinarias, que desarrollen temas especializados" (Macias, 2013:721).

Dicho lo anterior, los grupos de investigación, publicaciones, eventos contables y formación en maestrías y doctorados, son los factores que se deben impulsar en el contexto mundial para dinamizar esta corriente de investigación contable. Así se lograría mostrar resultados con mayor impacto en la academia y la práctica contable.

\section{III.3 Contable crítica}

Esta corriente considera los elementos de la teoría crítica, según argumentos del profesor Giraldo (2008:80), "presenta sus orígenes en los planteamientos de la escuela de Frankfurt y de Alfred Schutz, respectivamente, es posible hoy contar con una amplia literatura para realizar investigación alternativa a los fenómenos contables".

A esto se añade que la corriente crítica se fundamenta en distintas disciplinas como la sociología, la economía política, la ecología y la filosofía que estudiadas desde un enfoque interdisciplinario permiten el avance de las perspectivas críticas en contabilidad (Gómez, 2004).

Con base en fundamentos de la teoría crítica se formuló en contabilidad, una corriente de investigación denominada teoría contable crítica, con fundamentos en la teoría crítica de Habermas, Bourdieu, Giddens, Foucault y Marx y seguidores de la escuela de Francfort. Esta corriente se origina en Europa, específicamente "Reino Unido es la cuna de la investigación contable crítica, allí se ha identificado la necesidad de trascender las fronteras geográficas y culturales, para desarrollar debates con otros, Macias (2016:18) y se extiende a nivel internacional. 
Para el desarrollo de la investigación contable crítica (ICC), se recurre a un pluralismo metodológico (estudios de caso, investigación documental, etnometodología, entre otros) para el avance disciplinar, según los profesores Ryan; Scapens y Theobald (2004:62) "los enfoques metodológicos usados en la investigación contable, y en especial de la insistencia en que el llamado método científico no debe considerarse el enfoque único e ideal de toda gama de métodos de investigación", entonces se requiere impulsar nuevos caminos y tendencias que llevan al conocimiento y desarrollo de la disciplina contable.

De igual forma, el profesor Macias (2016:1) afirma que "la pluralidad de bases teóricas y metodológicas, en la literatura se ha dado en llamar a ese conjunto de trabajos "estudios críticos", desarrollados por todo un "movimiento contable crítico", y "la misión fundamental de la teoría contable crítica es generar capacidad de cuestionar la legitimidad política de los diversos regímenes de contabilidad"... (Macias, 2016:2). Entonces, el objetivo de la ICC es plantear preguntas sobre el uso de la contabilidad, más allá de implementarla (preparar reportes financieros) sin ningún cuestionamiento.

El fin de la ICC es promover un nuevo papel para la contabilidad como disciplina de conocimiento y vinculada con la crítica social y "es entendida como una parte que constituye y que es constituida por los macro conflictos entre las diferentes clases (p. ej, capitalista/ directivo vs. trabajador, el Estado vs. las corporaciones multinacionales" (Chua, 1986:64).

A esto se añade que la relación entre la teoría y práctica contable busca analizar críticamente la acción para generar cambios y transformaciones del orden social; sobre estas dinámicas autores como Chua, Tinker, Merino, Neimark y Cooper han realizado investigaciones desde esta corriente para identificar y eliminar las prácticas dominantes e ideológicas; hechos que generan reflexiones, tensiones, cuestionamientos y posibilidades en la investigación contable crítica.

Entonces, esos procesos generan controversias, al plantear diferentes significados, enfoques objetivo y subjetivo del ser humano, frente a condiciones sociales y materiales de dominación, como afirma Giraldo (2008:87) "bajo la óptica crítica se pone atención a las funciones económicas desarrolladas por la empresa capitalista (asignación de recursos, monitoreo, jerarquía y eficiencia), así la contabilidad se involucra en el control social y los conflictos entre personas".

Con todo lo anterior, se afirma que la contabilidad se enfoca más allá de una actividad netamente financiera y controladora de recursos limitados y pasa a analizar problemas macro económicos, de tipo social y humanos, como tasas de desempleo, equidad y redistribución de la riqueza.

Al respecto los planteamientos de los profesores, Arrington \& Puxty (1991, citados por Macias, 2016:6), establecen que la ICC propuso la teoría de la acción comunicativa de Habermas para la agenda de la ICC.

La contabilidad se situó en el contexto de los intereses humanos, con todas sus implicaciones. La contribución fue sintetizada al final del artículo en un boceto de siete 
puntos: (1) la contabilidad es una forma de acción moral que refleja las necesidades y deseos humanos; (2) la relación de la contabilidad con los intereses humanos es problemática; (3) el modelo de Habermas tiene tres mundos; (4) los actos contables siempre tienen consecuencias; (5) los individuos deben superar sus necesidades y deseos subjetivos; (6) la noción de validez no es estable en el mundo post-industrial en el que opera la contabilidad y (7) la alternativa a la racionalidad comunicativa es la coordinación de la acción social.

Para ilustrar los puntos 1 y 4 (acción moral de la contabilidad y consecuencias de los actos contables) se referencia un caso de manipulación en contabilidad:

Las dramáticas consecuencias del caso Enron, cuyo origen se sitúa en las manipulaciones contables, llevada a cabo por varios de sus directivos, puso nuevamente sobre la mesa la tesis mantenida por varios autores, asiduos colaboradores de revistas críticas, de que la contabilidad determina la vida de mucha gente (Archel, 2007:13).

Entonces, como se afirmó la contabilidad no solo provee información financiera para que los mercados operen, las empresas permanezcan y el estado funcione (Gómez, 2017) sino que influye en decisiones que afectan el interés público y la calidad de vida de las personas e impacto en el tejido social.

\section{CATEGORIZACIÓN DE LAS CORRIENTES DE INVESTIGACIÓN EN CONTABILIDAD}

Una vez descritos los principales fundamentos de las corrientes de investigación contable, se propone una matriz enfocado a contrastar 6 categorías de análisis. Estas se presentan por: origen, definición, propósito, enfoque científico, metodología e instrumento, como puntos de análisis en las tres corrientes de la disciplina contable: principal, crítica e interpretativa con el fin de contar con un paralelo que provoque su profundización.

Tabla No. 1. Corrientes en contabilidad y sus categorías principales

\begin{tabular}{|c|c|c|c|}
\hline Categoría & Principal & Interpretativa & Crítica \\
\hline Origen & $\begin{array}{l}\text { Inicios de la contabilidad } \\
\text { por partida doble (siglo } \\
\text { XV) } \\
\text { Se funda en la escuela } \\
\text { anglosajona y es un } \\
\text { sistema de información } \\
\text { patrimonialista - } \\
\text { financiera para la } \\
\text { toma de decisiones y } \\
\text { acumulación de riqueza. } \\
\text { Promoción del } \\
\text { pensamiento único. }\end{array}$ & $\begin{array}{l}\text { Surgimiento a } \\
\text { mediados del siglo XX. } \\
\text { Fundamentada en raíces } \\
\text { germanas y la escuela } \\
\text { europea (latina), para la } \\
\text { interpretación y } \\
\text { comprensión en las } \\
\text { ciencias sociales. } \\
\text { Promoción del } \\
\text { pensamiento alternativo. }\end{array}$ & $\begin{array}{l}\text { Surgimiento a mediados } \\
\text { del siglo XX. } \\
\text { Tiene su origen en raíces } \\
\text { germanas e interés por la } \\
\text { transformación social. } \\
\text { Fundamentada en } \\
\text { la escuela europea } \\
\text { (latina). Promoción del } \\
\text { pensamiento crítico. }\end{array}$ \\
\hline
\end{tabular}




\begin{tabular}{|c|c|c|c|}
\hline Definición & $\begin{array}{l}\text { Muestra una visión } \\
\text { simplificada de la } \\
\text { contabilidad, se } \\
\text { considera como técnica } \\
\text { de registro y control, } \\
\text { muestra el conocimiento } \\
\text { contable con sentido } \\
\text { pragmático y utilitarista. } \\
\text { Se presenta con la } \\
\text { regulación (usgaap, niif) } \\
\end{array}$ & $\begin{array}{l}\text { Formula una visión } \\
\text { amplia, incluyendo } \\
\text { aspectos de la realidad } \\
\text { física y social. Presenta } \\
\text { el conocimiento en } \\
\text { perspectiva teórica y } \\
\text { práctica. }\end{array}$ & $\begin{array}{l}\text { Exhibe un análisis } \\
\text { fustigador y cuestiona } \\
\text { la legitimidad política de } \\
\text { los diversos regímenes de } \\
\text { contabilidad para generar } \\
\text { propuestas de cambio y } \\
\text { transformación. }\end{array}$ \\
\hline Propósito & $\begin{array}{l}\text { Se ocupa principalmente } \\
\text { del funcionamiento de la } \\
\text { contabilidad, mediante } \\
\text { el reconocimiento, } \\
\text { medición, representación } \\
\text { y revelación para la } \\
\text { preparación de estados } \\
\text { financieros útiles en } \\
\text { la toma de decisiones } \\
\text { económicas. }\end{array}$ & $\begin{array}{l}\text { Busca describir, } \\
\text { comprender e } \\
\text { interpretar los } \\
\text { significados que los } \\
\text { actores humanos aplican } \\
\text { a los símbolos y a las } \\
\text { estructuras, así como la } \\
\text { naturaleza social de las } \\
\text { prácticas contables. }\end{array}$ & $\begin{array}{l}\text { Plantea preguntas sobre el } \\
\text { uso de la contabilidad, más } \\
\text { allá de implementarla sin } \\
\text { ningún cuestionamiento, } \\
\text { frente a los diversos } \\
\text { regímenes de contabilidad. }\end{array}$ \\
\hline $\begin{array}{l}\text { Enfoque } \\
\text { científico }\end{array}$ & $\begin{array}{l}\text { Respectivo a las ciencias } \\
\text { económicas, vinculado } \\
\text { con la globalización } \\
\text { económico - financiera } \\
\text { y concentración de la } \\
\text { riqueza. }\end{array}$ & $\begin{array}{l}\text { Relacionado con ciencias } \\
\text { sociales vinculado al ser } \\
\text { humano y dimensiones } \\
\text { económico, política, } \\
\text { social y ambiental. }\end{array}$ & $\begin{array}{l}\text { Relacionado con } \\
\text { las ciencias sociales } \\
\text { vinculado al ser humano y } \\
\text { dimensiones económico, } \\
\text { política, ambiental } \\
\text { y el interés para la } \\
\text { transformación social. }\end{array}$ \\
\hline Metodología & $\begin{array}{l}\text { Se prefieren métodos } \\
\text { cuantitativos de } \\
\text { recolección de datos } \\
\text { y aborda el enfoque } \\
\text { positivista y la } \\
\text { observación empírica. }\end{array}$ & $\begin{array}{l}\text { Utiliza el enfoque } \\
\text { cualitativo, donde } \\
\text { desarrolla una relación } \\
\text { cercana entre teoría } \\
\text { y práctica: revela que } \\
\text { hacen las personas } \\
\text { mientras interactúan } \\
\text { y hablan, su objetivo } \\
\text { es comprender las } \\
\text { acciones en sus } \\
\text { entornos naturales y } \\
\text { organizacionales. }\end{array}$ & $\begin{array}{l}\text { Enfoque cuantitativo y } \\
\text { cualitativo, poseen una } \\
\text { mezcla de subjetivismo y } \\
\text { objetivismo. } \\
\text { Cuenta con un aparato } \\
\text { teórico previo vinculado } \\
\text { con la hermenéutica } \\
\text { y dialéctica (Marx, } \\
\text { Habbermans). }\end{array}$ \\
\hline Instrumento & $\begin{array}{l}\text { Encuesta } \\
\text { Análisis bibliométrico. }\end{array}$ & $\begin{array}{l}\text { Utiliza el trabajo } \\
\text { etnográfico, el estudio } \\
\text { de caso y observación } \\
\text { participante, aplica } \\
\text { la entrevista, historia } \\
\text { oral, investigación } \\
\text { documental, } \\
\text { etnometodología y } \\
\text { grupos de discusión. }\end{array}$ & $\begin{array}{l}\text { Entrevista } \\
\text { trabajo etnográfico } \\
\text { estudio de caso } \\
\text { observación - participante } \\
\text { historia oral } \\
\text { investigación documental } \\
\text { etnometodología } \\
\text { teoría fundada y grupos de } \\
\text { discusión. }\end{array}$ \\
\hline
\end{tabular}

Fuente: Elaboración propia a partir de Archel (2007), Baker \& Owsen (2002), Giraldo (2008), Gómez (2004), Macias (2013, 2016), Ryan; Scapens y Theobald (2004). 
De las anteriores categorías presentadas en la tabla se sintetiza el propósito de las corrientes de investigación en contabilidad. En cuanto a la principal (funcionalista) se entiende a la contabilidad como sistema de información, con el fin de informar sobre la realidad económica de las organizaciones y fortalecer el modus operandi del sistema capitalista, de las instituciones que lo respaldan (B.M., F.M.I., O.M.C., O.C.D.E., G-20, entre otras) y de las empresas transnacionales (ETN), en un marco social y político para la concentración del capital, como afirma Rueda (2010:162), "un enfoque de mercado imperante, neoliberal, que mediante mensajes contables estandarizados, permite reducir el riego de inversión internacional".

Con respecto al propósito de la corriente contable interpretativa esta analiza la naturaleza de aspectos sociales vinculados con práctica contable en el accionar humano en el uso y exploración de recursos económicos y ambientales, al respecto Larrinaga (1999:114) plantea "el significado que tienen las prácticas contables, es el resultado de la interacción social, por lo que la información o lenguaje contable pueden tener diferentes significados en función de los contextos sociales, políticos e históricos".

Por su parte, la corriente contable crítica tiene un propósito que va más allá al formular interrogantes y cuestionar los regímenes contables existentes que favorecen el mercado y dejan atrás al ser humano, sus perspectivas sociales y ambientales, como lo expresan Gómez y Opina (2009:14) "estas perspectivas buscan nutrir la reflexión con una comprensión del rol social, institucional y político de la contabilidad”, con el fin de promover cambios en el bienestar y desarrollo a escala humana, para lo cual se requiere de la transformación social, donde se elimine la dominación ideológica, promueva la justicia y equidad.

\section{CONCLUSIONES}

El interés de este artículo fue contribuir al estudio de las corrientes de investigación en contabilidad a partir de la revisión documental y fundamentos sobre estas temáticas, así se analizó como la corriente principal de la contabilidad (funcionalista) está interesada en "representar" la realidad económica de las entidades en beneficio del mercado y los grandes capitales.

1. La demanda de información contable que maximice beneficios económicos, permite a ciertos grupos de interés (instituciones internacionales y corporaciones) que se adopte un modelo global con enfoque financiero, "En este escenario se requiere de sistemas de información, que bajo estándares mundialmente aceptados brinden información útil que facilite las decisiones de inversión, financiación, compraventa y se promueva la integración económica internacional" (Castillo; Córdoba y Villarreal, 2014:123); promovido por instituciones de carácter mundial (IASB-IFAC), al determinar un propósito utilitarista y de pensamiento único en contabilidad.

2. Si bien es cierto que predomina la corriente principal (funcionalista), desde hace más de cuatro décadas se viene cuestionando su legitimidad; así la contabilidad no es reflejo automático de asuntos económicos, por el contrario, responde a condiciones his- 
tóricas, e intereses ideológicos y políticos de distintos grupos empresariales, estatales, académicos y sociales. Todo esto en conjunto, es un llamado a la comunidad académica a estudiar a profundidad la corriente contable principal con el fin de analizar su lógica y limitaciones.

3. Se identificó que el enfoque científico de la corriente principal se vincula con las ciencias económicas, mientras que la corriente interpretativa y crítica, se relaciona con las ciencias sociales; por eso, en el primero predomina el método con un enfoque cuantitativo; mientras que las últimas, enfatiza en el método con enfoque cualitativo. Dicho lo anterior, se debe profundizar en el diseño, validación y aplicación de los instrumentos utilizados en cada corriente de investigación como una invitación para continuar reflexionado sobre este tema.

4. La investigación contable interpretativa y crítica busca centrase en el desarrollo del ser humano y sus distintas dimensiones sociales, políticas y ambientales. La misión de la corriente interpretativa es darle sentido a la práctica contable que incluya análisis de su teoría y praxis. Por su parte, la corriente crítica, discute la legitimidad política del régimen de la información financiera (NIIF), además se interesa por la transformación social, y cuestiona el modelo económico imperante (capitalismo), para poner atención en el ser humano, la vida, equidad en la distribución de la riqueza, justicia y promoción del desarrollo sostenible.

\section{REFERENCIAS}

(1) ARRINGTON, C. E., \& PUXTY, A. G (1991). "Accounting, interests, and rationality: a communicative relation". En: Crítical Perspectives on Accounting, Vol. 2, No. 1, pp. 31-58.

(2) ARCHEL D., Pablo (2007). Teoría e investigación crítica en contabilidad. Un estudio de caso. Asociación Española de Contabilidad y Administración -AECA. Madrid, España. Pp. 145.

(3) BAKER, Richard; BETTNER, Mark (1997). Interpretive and crítical research in accounting: a commentary on its absence from mainstream accounting research. Crítical Perspectives on Accounting. En: Crítical Perspectives on Accounting. Vol. 8, No. 4. Pp. 293 - 310.

(4) CASTILlO M., Carlos; CÓRDOBA M., Jorge; VILLARREAL, José (2008). “Caracterización del pensamiento contable: desarrollo y retos”. En: Revista Management, julio-diciembre 2008/Vol. XVII, No. 30. Bogotá, Colombia. Pp. 17-31.

(5) CASTILlO M., Carlos; CÓRDOBA M., Jorge; VILLARREAL, José (2014)."Estándares internacionales de educación (IES) en contabilidad y aseguramiento: nuevos retos de la profesión contable". En: Tendencias. Revista de la Facultad de Ciencias Económicas y Administrativas. Julio-Diciembre, 2014/Vol. XV, No. 2. Bogotá, Colombia. Pp. 118-135.

(6) CHUA, Wai Fong (1986). Desarrollos radicales en el pensamiento contable. En: Avances interdisciplinarios para una comprensión crítica de la contabilidad. Textos paradigmáticos de las corrientes heterodoxas. 
Escuela de Administración y Contaduría Pública - Universidad Nacional de Colombia. Departamento de Ciencias Contables - Universidad de Antioquia. Pp. 37-75.

(7) FRANCO R., Rafael (2015). “Dimensión heterotopológica de la regulación contable. Perspectivas críticas sobre gobierno corporativo y regulación”. En: Revista Científica General Jose Maria Cordova, julio-diciembre, 2015/Vol. 13, No. 16. Bogotá, Colombia. Pp. 173-199.

(8) GIRALDO G., Gregorio (2008). Repercusión de la teoría crítica en la investigación contable. En: Perspectivas críticas de la contabilidad. "reflexiones y críticas alternas al pensamiento único. Memorias del VII Simposio Nacional de Investigación Contable y Docencia. 6-8 Junio de 2008. Universidad nacional de Colombia-Centro Colombiano de Investigaciones Contables. Bogotá, Colombia. Pp. 77-94.

(9) GIRALDO G., Gregorio; FLOR O., Naila K (2015). La contabilidad: un dispositivo de control del capitalismo financiero, Memorias Jornada Internacional de Estudios Disciplinares en Contabilidad, Universidad Militar Nueva Granada, agosto, 2015/Vol. I. Bogotá, Colombia Pp. 446-454.

(10) GÓMEZ V, Mauricio (2004). "Breve introducción al estado del arte de la orientación crítica en la disciplina contable”. En: Revista Contaduría. Universidad de Antioquia. Julio-Diciembre, 2004. No. 45. Medellín, Colombia. Pp. 113-132.

(11) GÓMEZ V, Mauricio (2016). NIIF y MIPYMES: los retos de la contabilidad para el contexto y la productividad. Escuela de Administración y Contaduría Pública EACP - Centro de investigaciones para el desarrollo CID. Junio, 2016/No. 25. Bogotá, Colombia. Pp. 34

(12) GÓMEZ V, Mauricio; OSPINA Z, Carlos M. (2009). Avances interdisciplinarios para una comprensión crítica de la contabilidad. Textos paradigmáticos de la corrientes heterodoxas. Escuela de Administración y Contaduría Pública - Universidad Nacional de Colombia. Departamento de Ciencias Contables - Universidad de Antioquia.

(13) JURADO S., Jorge A (2015). Lo lóbrego del control de gestión. Memorias Jornada Internacional de Estudios Disciplinares en Contabilidad, Universidad Militar Nueva Granada, agosto, 2015/Vol. I. Bogotá, Colombia Pp. 470-477.

(14) LARRINAGA G., Carlos (1999). "Perspectivas alternas de investigación en contabilidad: una revisión”. En: revista de contabilidad, Enero-Junio, 1999/Vol. 2, No. 3. Santander, España. Pp. 103-131.

(15) MACÍAS, Hugo A (2013). "Vínculos de la investigación contable interpretativa con la producción académica colombiana: avances y oportunidades". En: Cuadernos de Contabilidad, Julio-Diciembre, 2013/Vol. 14, No. 35. Bogotá, Colombia. Pp. 699-727.

(16) MACÍAS, Hugo (2016). "Introducción a la investigación contable crítica (icc) en su contexto original”. En: tercer congreso global en contabilidad y finanzas - INTERGES. Contribuciones a la perspectiva crítica en Contabilidad, Finanzas y Gestión. Universidad Nacional de Colombia, Bogotá, Colombia. 23 al 25 de agosto de 2016. Pp. 28.

(17) MACHADO R., Marco; VILORIA O., Norka (2016). Los caminos de la teoría contable: de las descripciones a la construcción de pensamiento. En: Caminos contables. Problemas y metodologías para el desarrollo de la investigación. Departamento de Ciencias Contables Universidad de Antioquia - Grupo de Investigación y Consultorias Contables-GICCO. Sello editorial Centro de Investigaciones y Consultorias - CIC. Pp. 268-311.

(18) RYAN, Bod; SCAPENS, Robert; THEOBALD, Michael (2004). Metodología de la investigación en Finanzas y Contabilidad. Ediciones Deusto. Barcelona, España. Pp. 319.

(19) RUEDA D, Gabriel (2010). "El papel de la contabilidad ante la actual realidad económica, social y política del país. Más allá de la convergencia de prácticas mundiales". En: Cuadernos de Contabilidad, Enero-Junio, 2010/Vol. 11, No. 28. Bogotá, Colombia. Pp. 149-169.

(20) SALGADO C, Jorge (2005). “Lo lóbrego del control de gestión”. En: Memorias I jornada internacional de estudios disciplinares en contabilidad y I encuentro de estudiantes de contaduría pública. Universidad Militar Nueva Granada 27 y 28 de agosto de 2005. Pp. 470-477. 